IJMMS 29:12 (2002) 701-718

PII. S0161171202020197

http://ijmms.hindawi.com

(c) Hindawi Publishing Corp.

\title{
A GENERALISED HOPF ALGEBRA FOR SOLITONS
}

\author{
FALLEH R. AL-SOLAMY and EDWIN J. BEGGS
}

Received 17 April 2001 and in revised form 22 October 2001

\begin{abstract}
This paper considers a generalisation of the idea of a Hopf algebra in which a commutative ring replaces the field in the unit and counit. It is motivated by an example from the inverse scattering formalism for solitons. We begin with the corresponding idea for groups, where the concept of the identity is altered.
\end{abstract}

2000 Mathematics Subject Classification: 16W30, 37K30.

1. Introduction. Group factorisation plays a vital role in the inverse scattering procedure $[1,8]$. For example, the Riemann-Hilbert problem is a (not quite exact) factorisation of group-valued functions on the real line into functions analytic on the lower half-plane times functions analytic on the upper half-plane. However, there is a problem, a group-valued function which is analytic on the lower half-plane need not have an inverse which is analytic there. On the Lie algebra level all is well since any smooth loop which is uniformly sufficiently close to the identity and is analytic on the lower half-plane has an inverse which is also analytic on the lower half-plane. To avoid the problem, we consider the Lie algebras or a neighbourhood of a group near the identity. This corresponds in inverse scattering to considering solutions not too far from the vacuum. However, the soliton solutions for many integrable systems are characterized by meromorphic loops, and there the factors are very definitely not closed under inverse. For example, if we take the meromorphic function given by (for $P \in M_{n}(\mathbb{C})$ a Hermitian projection matrix and $P^{\perp}=1-P$ )

$$
\phi(\lambda)=P^{\perp}+\frac{\lambda-\bar{\alpha}}{\lambda-\alpha} P
$$

which has a pole at $\alpha$ in the upper half-plane, its inverse is given by

$$
\phi^{-1}(\lambda)=P^{\perp}+\frac{\lambda-\alpha}{\lambda-\bar{\alpha}} P
$$

which has a pole at $\bar{\alpha}$ in the lower half-plane.

The meromorphic loops which specify the solitons in a classical integrable system are not uniquely defined, there are vacuum loops which can be added without generating any extra solitons. However, these can be thought of as allowing soliton-antisoliton pair creation in the integrable system. When the system is quantised a vacuum loop could be perturbed into a soliton-antisoliton pair by a slight movement of the pole positions. Effectively the vacuum loops get round the problem which would occur 
if we could count solitons by the number of poles. The number of poles cannot be changed by a small perturbation, so without the vacuum loops soliton-antisoliton pair production might seem impossible. To calculate the total quantum energy and momentum for a soliton, the contributions for these vacuum loops would have to be added. This quantum correction is observed in the Sine-Gordon model, calculated by other methods [11].

The existence of the vacuum loops and the fact that the upper and lower factors for the meromorphic loop group are not groups are related, and are both taken into account in the ideas described in this paper of an almost group and matched pairs of almost groups. This naturally leads to the idea of an almost Hopf algebra, in which the unit and counit map are modified to use a commutative algebra instead of the ground field. From the discussion above, the commutative algebra would arise from the vacuum loops. A group factorisation into a subgroup (a group doublecross product) is well known to lead to a Hopf algebra bicrossproduct [2, 5, 10]. Here, we also carry out the corresponding procedure for almost groups and almost Hopf algebras.

This is not the only generalisation of the idea of a Hopf algebra. In [3], there are axioms for weak $C^{*}$-Hopf algebras, but in this case the unit and counit are not algebra maps, which they are in our axioms.

Note that although we use some infinite examples of almost groups as motivation, in the detailed proofs of the results about the algebras we always assume that almost groups are finite.

\section{Almost groups}

DEFINITION 2.1. An almost group is a set $G$ with an associative binary operation ·, a 1-1 correspondence $i: G \rightarrow G$ (written $g \mapsto g^{i}$ ), and a set $J \subset G$ which is closed under the binary operation $\cdot$ and $i$. Also the following properties are satisfied:

(i) $(g h)^{i}=h^{i} g^{i}$, for all $g, h \in G$;

(ii) for all $g \in G$, and for all $j \in J, j g=g j$;

(iii) for all $g \in G, g^{i}=g^{i} g \in J$;

(iv) $\left(g^{i}\right)^{i}=g$, for all $g \in G$.

EXAMPLE 2.2. In the case where $J=\{e\}$, where $g e=g$ for all $g \in G$, we just get a group.

EXAMPLE 2.3. Take $G$ to be the set of meromorphic functions from $\mathbb{C}_{\infty}$ to $\mathrm{GL}_{n}(\mathbb{C})$ which are unitary on the real axis, normalized to the identity at infinity, and have all poles in the upper half-plane. All such loops can be factored as a product of functions of the form (1.1). We define the $i$ operation on (1.1) by

$$
\phi^{i}(\lambda)=\frac{\lambda-\bar{\alpha}}{\lambda-\alpha} P^{\perp}+P
$$

Here $G$ is an almost group when $J$ consists of all meromorphic complex-valued functions times the identity matrix (the vacuum loops), and the binary operation is the usual matrix multiplication. 
EXAMPLE 2.4 (Milnor's construction of the total space of the classifying bundle of a group [6, 7, 9]). Suppose that we have an abelian group $A$ and a topological space $E A$ consisting of step functions: $[0,1) \rightarrow A \times \mathbb{N}$ which are constant on the intervals $\left[x_{i}, x_{i+1}\right)$ where $0=x_{0}<x_{1}<\cdots<x_{N}=1$, and where the $\mathbb{N}$-valued numbers are strictly increasing in successive intervals.

The multiplication on $E A$ is defined by pointwise multiplication of the abelian group elements and adding the numbers, and the $i$ operation is defined by taking the inverse of the abelian group elements without any change in the numbers.

Then we can say that $E A$ is an almost group if we take $J$ to consist of steps all taking value the identity element in $A$, but with different numbers.

EXAMPLE 2.5. Suppose that $G=\{a, b, c\}$ and $J=\{a, b\}$. We define the multiplication and the $i$ operation by $x \cdot y=a$ and $x^{i}=x$ for all $x, y \in G$.

Alternatively we could take the case where $a \cdot x=x \cdot a=x$ for all $x \in G$, and the other products are equal to $b$, and the $i$ operation is as defined before.

EXAMPLE 2.6. Consider $G=A \times A$ where $A$ is an abelian group, with multiplication $(a, b) \cdot(c, d)=(a c, b d)$, the $i$ operation $(a, b)^{i}=(b, a)$ and $J=\{(a, a): a \in A\}$.

EXAMPLE 2.7. A Clifford semigroup is an example of an almost group, where $J$ consists of idempotents [4].

3. Almost Hopf algebras. In this section, and throughout the rest of the paper, we assume that $k$ is a ground field, and all algebras and maps are taken over $k$. Now we are in the position to give a definition for an almost Hopf algebra $H$ which has the same rules for Hopf algebra $H$ except $\epsilon: H \rightarrow H_{J}$ and $\eta: H_{J} \rightarrow H$ where $H_{J} \subset H$.

Definition 3.1. An almost Hopf algebra $\left(H,+, \cdot, \eta, \Delta, \epsilon, S ; H_{J}\right)$ is an associative algebra $H$ with the following additional structure:

(i) $H_{J}$ is a commutative associative algebra.

(ii) A coassociative comultiplication map $\Delta: H \rightarrow H \otimes H$.

(iii) A map $\epsilon: H \rightarrow H_{J}$ satisfying $(\epsilon \otimes i d) \Delta(h)=\tau((i d \otimes \epsilon) \Delta(h))$ for all $h \in H$, where $\tau(h \otimes j)=j \otimes h$.

(iv) A map $\eta: H_{J} \rightarrow H$ satisfying $\cdot(\eta \otimes i d)=\cdot(i d \otimes \eta) \tau: H_{J} \otimes H \rightarrow H$.

(v) A linear antipode map $S: H \rightarrow H$ obeying $\cdot(S \otimes i d) \circ \Delta(h)=\cdot(i d \otimes S) \circ \Delta(h)=$ $\eta \circ \epsilon(h)$ for all $h \in H$.

(vi) The maps $\Delta, \epsilon$, and $\eta$ are algebra maps.

If $G$ were a finite group, then its group algebra $k G$ and its function algebra $k(G)$ would be Hopf algebras. We need to check that the same is true for almost groups and almost Hopf algebras.

Note that we do not assume that $\cdot(\epsilon \otimes i d) \Delta(h)=h$, and this distinguishes this definition from that of a Hopf algebra over a ring. For example, this equality does not work for $k G$, for $(G, J)$ a finite almost group (see Example 3.3).

EXAMPLE 3.2. Let $(G, J)$ be a finite almost group. Take a basis of $k(G)$ consisting of elements of the form $\delta_{x}$ for $x \in G$, and a basis for $k(G)_{J}=k(J)$ of the form $\delta_{j}$ for 
$j \in J$. The operations are defined as

$$
\begin{gathered}
\delta_{x} \cdot \delta_{y}=\delta_{x} \delta_{x, y}, \quad \Delta\left(\delta_{x}\right)=\sum_{x=y z} \delta_{y} \otimes \delta_{z}, \\
S\left(\delta_{x}\right)=\delta_{x^{i}}, \quad \epsilon\left(\delta_{x}\right)=\left\{\begin{array}{ll}
0, & \text { if } x \notin J, \\
\delta_{x}, & \text { if } x \in J,
\end{array} \quad \eta\left(\delta_{j}\right)=\sum_{z \in G: j=z z^{i}} \delta_{z} .\right.
\end{gathered}
$$

Here the symbol $\delta_{x, y}$ is the Kroneker delta, which is one if $x=y$ and zero otherwise. We will now check the rules of an almost Hopf algebra.

It is easy to check that - gives an associative multiplication and that $\Delta$ gives a coassociative comultiplication, that is, $(\Delta \otimes i d) \Delta\left(\delta_{x}\right)=(i d \otimes \Delta) \Delta\left(\delta_{x}\right)$. For Definition 3.1(iii) we get

$$
\begin{gathered}
(\epsilon \otimes i d) \Delta\left(\delta_{x}\right)=(\epsilon \otimes i d)\left(\sum_{x=y z} \delta_{y} \otimes \delta_{z}\right)=\sum_{x=j z: j \in J} \delta_{j} \otimes \delta_{z}, \\
\tau(i d \otimes \epsilon) \Delta\left(\delta_{x}\right)=\tau(i d \otimes \epsilon)\left(\sum_{x=z y} \delta_{z} \otimes \delta_{y}\right)=\tau\left(\sum_{x=z j: j \in J} \delta_{z} \otimes \delta_{j}\right),
\end{gathered}
$$

which are the same as $z j=j z$ for all $j \in J$. For Definition 3.1(iv),

$$
\begin{gathered}
\cdot(\eta \otimes i d)\left(\delta_{j} \otimes \delta_{x}\right)=\cdot\left(\sum_{j=z z^{i}} \delta_{z} \otimes \delta_{x}\right)=\sum_{j=z z^{i}} \delta_{z} \delta_{z, x}, \\
\cdot(i d \otimes \eta) \tau\left(\delta_{j} \otimes \delta_{x}\right)=\cdot(i d \otimes \eta)\left(\delta_{x} \otimes \delta_{j}\right)=\cdot\left(\delta_{x} \otimes \sum_{j=z z^{i}} \delta_{z}\right)=\sum_{j=z z^{i}} \delta_{x} \delta_{x, z} \cdot
\end{gathered}
$$

For Definition 3.1(v),

$$
\begin{aligned}
& \cdot(S \otimes i d) \Delta\left(\delta_{x}\right)=\cdot(S \otimes i d)\left(\sum_{x=y z} \delta_{y} \otimes \delta_{z}\right)=\cdot\left(\sum_{x=y z} \delta_{y^{i}} \otimes \delta_{z}\right)=\sum_{x=y y^{i}} \delta_{y^{i}}, \\
& \cdot(i d \otimes S) \Delta\left(\delta_{x}\right)=\cdot(i d \otimes S)\left(\sum_{x=y z} \delta_{y} \otimes \delta_{z}\right)=\cdot\left(\sum_{x=y z} \delta_{y} \otimes \delta_{z^{i}}\right)=\sum_{x=y y^{i}} \delta_{y},
\end{aligned}
$$

where we have used the fact that if $y=z^{i}$ then $y^{i}=\left(z^{i}\right)^{i}=z$ by Definition 2.1(iv). But these two expressions are the same, as can be seen by putting $w=y^{i}$ in the second and noting that $y y^{i}=y^{i} y$ by Definition 2.1(iii). Now note that the expressions give zero unless $x \in J$, as $y y^{i} \in J$, and then they have value $\eta\left(\epsilon\left(\delta_{x}\right)\right)$. It is left to the reader to check that $\Delta, \epsilon$, and $\eta$ are algebra maps.

EXAMPLE 3.3. Let $(G, J)$ be a finite almost group. The almost group algebra has $H=k G$ and $H_{J}=k J$, with multiplication given by the usual linear extension of the almost group multiplication. The operations are (for $x \in G$ and $j \in J) \Delta(x)=x \otimes x$, $S(x)=x^{i}, \epsilon(x)=x x^{i}$, and $\eta(j)=j$. Then the rules for an almost Hopf algebra are satisfied. 
It is easy to check that - gives an associative multiplication and that $\Delta$ gives a coassociative comultiplication. For Definition 3.1(iii),

$$
\begin{aligned}
(\epsilon \otimes i d) \Delta(x) & =(\epsilon \otimes i d)(x \otimes x)=x x^{i} \otimes x, \\
\tau(i d \otimes \epsilon) \Delta(x) & =\tau(i d \otimes \epsilon)(x \otimes x)=\tau\left(x \otimes x x^{i}\right)=x x^{i} \otimes x .
\end{aligned}
$$

For Definition 3.1(iv),

$$
\begin{aligned}
\cdot(\eta \otimes i d)(j \otimes x) & =\cdot(j \otimes x)=j x, \\
\cdot(i d \otimes \eta) \tau(j \otimes x) & =\cdot(i d \otimes \eta)(x \otimes j)=\cdot(x \otimes j)=x j=j x,
\end{aligned}
$$

where we have used Definition 2.1(ii). For Definition 3.1(v),

$$
\begin{aligned}
& \cdot(S \otimes i d) \Delta(x)=\cdot(S \otimes i d)(x \otimes x)=\cdot\left(x^{i} \otimes x\right)=x^{i} x, \\
& \cdot(i d \otimes S) \Delta(x)=\cdot(i d \otimes S)(x \otimes x)=\cdot\left(x \otimes x^{i}\right)=x x^{i}=x^{i} x,
\end{aligned}
$$

where we have used Definition 2.1(iii), so both of these expressions are equal to $\eta(\epsilon(x))$. It is easy to see that $\Delta$ and $\eta$ are algebra maps. For $\epsilon$,

$$
\epsilon(x) \epsilon(y)=x x^{i} y y^{i}=x y y^{i} x^{i}=x y(x y)^{i}=\epsilon(x y),
$$

where we have used Definition 2.1(i) and (ii).

Proposition 3.4. In Examples 3.2 and 3.3, $S$ is an antialgebra map, that is, $S\left(h h^{\prime}\right)=$ $S\left(h^{\prime}\right) S(h)$.

Proof. This is immediate in both cases.

\section{Matched pairs and doublecross products}

DEFINITION 4.1 (Matched pairs of almost groups). Suppose that $\left(G, J_{G}\right)$ and $\left(M, J_{M}\right)$ are almost groups. Now take functions $\triangleright: M \times G \rightarrow G$, and $\triangleleft: M \times G \rightarrow M$, which obey the following rules, for all $s, t \in M$, and $u, v \in G$ :

$$
\begin{aligned}
& s \triangleleft u v=(s \triangleleft u) \triangleleft v, \quad s t \triangleleft u=(s \triangleleft(t \triangleright u))(t \triangleleft u), \\
& s t \triangleright u=s \triangleright(t \triangleright u), \quad s \triangleright u v=(s \triangleright u)((s \triangleleft u) \triangleright v) \text {. }
\end{aligned}
$$

With the $i$ operations we require

$$
(s \triangleleft u)^{i} \triangleright(s \triangleright u)^{i}=u^{i}, \quad(s \triangleleft u)^{i} \triangleleft(s \triangleright u)^{i}=s^{i},
$$

and also, for all $j \in J_{G}$ or $J_{M}$, we have

$$
j \triangleright u=u, \quad j \triangleleft u=j, \quad s \triangleright j=j, \quad s \triangleleft j=s .
$$

Proposition 4.2. Given a matched pair $\left(G, J_{G}\right)$ and $\left(M, J_{M}\right)$ of almost groups we can construct a doublecross product almost group $G \bowtie M$, which consists of the set $G \times M$ with binary operation $(u, s)(v, t)=(u(s \triangleright v),(s \triangleleft v) t)$ and $(u, s)^{i}=\left(s^{i} \triangleright u^{i}, s^{i} \triangleleft u^{i}\right)$. Finally we set $J_{G \bowtie M}=J_{G} \times J_{M}$. 
Proof. The proof that the multiplication is associative is tedious but straightforward. For Definition 2.1(i),

$$
\begin{aligned}
((u, s)(v, t))^{i} & =(u(s \triangleright v),(s \triangleleft v) t)^{i} \\
& =\left(t^{i}(s \triangleleft v)^{i} \triangleright(s \triangleright v)^{i} u^{i}, t^{i}(s \triangleleft v)^{i} \triangleleft(s \triangleright v)^{i} u^{i}\right) \\
& =\left(t^{i} \triangleright v^{i}\left(s^{i} \triangleright u^{i}\right),\left(t^{i} \triangleleft v^{i}\right) s^{i} \triangleleft u^{i}\right), \\
(v, t)^{i}(u, s)^{i} & =\left(t^{i} \triangleright v^{i}, t^{i} \triangleleft v^{i}\right)\left(s^{i} \triangleright u^{i}, s^{i} \triangleleft u^{i}\right),
\end{aligned}
$$

and these can be seen to be the same after the application of the product rule. For Definition 2.1(ii), given $j \in J_{G}$ and $n \subset J_{M}$,

$$
\begin{aligned}
& (j, n)(u, s)=(j(n \triangleright u),(n \triangleleft u) s)=(j u, n s), \\
& (u, s)(j, n)=(u(s \triangleright j),(s \triangleleft j) n)=(u j, s n) .
\end{aligned}
$$

For Definition 2.1(iii),

$$
\begin{aligned}
(u, s)(u, s)^{i} & =(u, s)\left(s^{i} \triangleright u^{i}, s^{i} \triangleleft u^{i}\right) \\
& =\left(u u^{i},\left(s \triangleleft\left(s^{i} \triangleright u^{i}\right)\right)\left(s^{i} \triangleleft u^{i}\right)\right) \\
& =\left(u u^{i}, s s^{i} \triangleleft u^{i}\right) \\
& =\left(u u^{i}, s s^{i}\right), \\
(u, s)^{i}(u, s) & =\left(s^{i} \triangleright u^{i}, s^{i} \triangleleft u^{i}\right)(u, s)=\left(\left(s^{i} \triangleright u^{i}\right)\left(\left(s^{i} \triangleleft u^{i}\right) \triangleright u\right), s^{i} s\right) \\
& =\left(s^{i} \triangleright u^{i} u, s^{i} s\right)=\left(u^{i} u, s^{i} s\right) .
\end{aligned}
$$

For Definition 2.1(iv),

$$
\begin{aligned}
\left((u, s)^{i}\right)^{i} & =\left(s^{i} \triangleright u^{i}, s^{i} \triangleleft u^{i}\right)^{i} \\
& =\left(\left(s^{i} \triangleleft u^{i}\right)^{i} \triangleright\left(s^{i} \triangleright u^{i}\right)^{i},\left(s^{i} \triangleleft u^{i}\right)^{i} \triangleleft\left(s^{i} \triangleright u^{i}\right)^{i}\right) \\
& =\left(\left(u^{i}\right)^{i},\left(s^{i}\right)^{i}\right) \\
& =(u, s) .
\end{aligned}
$$

DeFinITION 4.3 (Bicrossproduct almost Hopf algebras). From the finite matched pair $\left(G, J_{G}\right)$ and $\left(M, J_{M}\right)$ we define an almost Hopf algebra $H=k M \bowtie k(G)$ with basis $s \otimes \delta_{u}$ where $s \in M$ and $u \in G$. We take $H_{J}=k J_{M} \otimes k\left(J_{G}\right) \subset H$. Here $k M$ is the almost group almost Hopf algebra of $M$ and $k(G)$ is the almost Hopf algebra of functions on $G$. Explicit formulae for $k M \bowtie k(G)$ are as follows:

$$
\begin{gathered}
\left(s \otimes \delta_{u}\right)\left(t \otimes \delta_{v}\right)=\delta_{u, t \triangleright v}\left(s t \otimes \delta_{v}\right), \quad \Delta\left(s \otimes \delta_{u}\right)=\sum_{x y=u} s \otimes \delta_{x} \otimes s \triangleleft x \otimes \delta_{y}, \\
S\left(s \otimes \delta_{u}\right)=(s \triangleleft u)^{i} \otimes \delta_{(s \triangleright u)^{i}}, \quad \epsilon\left(s \otimes \delta_{u}\right)= \begin{cases}0, & \text { if } u \notin J_{G}, \\
s s^{i} \otimes \delta_{u}, & \text { if } u \in J_{G},\end{cases} \\
\eta\left(j \otimes \delta_{n}\right)=\sum_{n=z z^{i}} j \otimes \delta_{z}, \quad \forall j \in J_{M}, n \in J_{G} .
\end{gathered}
$$


Now we would like to check the rules for an almost Hopf algebra, but first we need to prove certain results.

Proposition 4.4. For all $s \in M$ and $w \in G,(s \triangleleft w)^{i}(s \triangleleft w)=s s^{i}=s^{i} s$.

Proof. From the rules for a matched pair,

$$
\begin{aligned}
(s \triangleleft w)^{i}(s \triangleleft w) & =(s \triangleleft w)^{i}(s \triangleleft w) \triangleleft w^{i} \\
& =\left((s \triangleleft w)^{i} \triangleleft\left((s \triangleleft w) \triangleright w^{i}\right)\right)\left((s \triangleleft w) \triangleleft w^{i}\right) \\
& =\left((s \triangleleft w)^{i} \triangleleft\left((s \triangleleft w) \triangleright w^{i}\right)\right)\left(s \triangleleft w w^{i}\right) \\
& =\left((s \triangleleft w)^{i} \triangleleft\left((s \triangleleft w) \triangleright w^{i}\right)\right) s .
\end{aligned}
$$

Now we know that, (where $(s \triangleright w)^{i}(s \triangleright w)=j$ )

$$
\begin{aligned}
s \triangleright w w^{i} & =(s \triangleright w)\left((s \triangleleft w) \triangleright w^{i}\right)=w w^{i}, \\
(s \triangleright w)^{i}(s \triangleright w)\left((s \triangleleft w) \triangleright w^{i}\right) & =(s \triangleright w)^{i} w w^{i}, \\
(s \triangleleft w)^{i} \triangleleft j\left((s \triangleleft w) \triangleright w^{i}\right) & =(s \triangleleft w)^{i} \triangleleft(s \triangleright w)^{i} w w^{i}, \\
(s \triangleleft w)^{i} \triangleleft\left((s \triangleleft w) \triangleright w^{i}\right) & =\left((s \triangleleft w)^{i} \triangleleft(s \triangleright w)^{i}\right) \triangleleft w w^{i} \\
& =s^{i} \triangleleft w w^{i} \\
& =s^{i} .
\end{aligned}
$$

Proposition 4.5. For all $s \in M$ and $w \in G,(s \triangleright w)(s \triangleright w)^{i}=w w^{i}=w^{i} w$.

PROoF. From the rules for a matched pair,

$$
\begin{aligned}
(s \triangleright w)(s \triangleright w)^{i} & =s^{i} \triangleright(s \triangleright w)(s \triangleright w)^{i} \\
& =\left(s^{i} \triangleright(s \triangleright w)\right)\left(\left(s^{i} \triangleleft(s \triangleright w)\right) \triangleright(s \triangleright w)^{i}\right) \\
& =\left(s^{i} s \triangleright w\right)\left(\left(s^{i} \triangleleft(s \triangleright w)\right) \triangleright(s \triangleright w)^{i}\right) \\
& =w\left(\left(s^{i} \triangleleft(s \triangleright w)\right) \triangleright(s \triangleright w)^{i}\right) .
\end{aligned}
$$

Now we know that, (where $\left.(s \triangleleft w)(s \triangleleft w)^{i}=j\right)$

$$
\begin{aligned}
s^{i} s \triangleleft w & =\left(s^{i} \triangleleft(s \triangleright w)\right)(s \triangleleft w)=s^{i} s \\
\left(s^{i} \triangleleft(s \triangleright w)\right)(s \triangleleft w)(s \triangleleft w)^{i} & =s^{i} s(s \triangleleft w)^{i} \\
\left(s^{i} \triangleleft(s \triangleright w)\right) j \triangleright(s \triangleright w)^{i} & =s^{i} s(s \triangleleft w)^{i} \triangleright(s \triangleright w)^{i} \\
\left(s^{i} \triangleleft(s \triangleright w)\right) \triangleright(s \triangleright w)^{i} & =s^{i} s \triangleright\left((s \triangleleft w)^{i} \triangleright(s \triangleright w)^{i}\right) \\
& =s^{i} s \triangleright w^{i} \\
& =w^{i} .
\end{aligned}
$$

Now we check that the construction in Definition 4.3 gives an almost Hopf algebra. It is fairly standard to check that the product is associative and that the coproduct is 
coassociative. For Definition 3.1(iii), where $j \in J_{G}$,

$$
\begin{aligned}
(\epsilon \otimes i d) \Delta\left(s \otimes \delta_{u}\right) & =(\epsilon \otimes i d)\left(\sum_{u=x y} s \otimes \delta_{x} \otimes s \triangleleft x \otimes \delta_{y}\right) \\
& =\sum_{u=j y} s s^{i} \otimes \delta_{j} \otimes s \triangleleft j \otimes \delta_{y}=\sum_{u=j y} s s^{i} \otimes \delta_{j} \otimes s \otimes \delta_{y}, \\
\tau(i d \otimes \epsilon) \Delta\left(s \otimes \delta_{u}\right) & =\tau(i d \otimes \epsilon)\left(\sum_{u=y x} s \otimes \delta_{y} \otimes s \triangleleft y \otimes \delta_{x}\right) \\
& =\tau\left(\sum_{u=y j} s \otimes \delta_{y} \otimes(s \triangleleft y)(s \triangleleft y)^{i} \otimes \delta_{j}\right) \\
& =\tau\left(\sum_{u=j y} s \otimes \delta_{y} \otimes s s^{i} \otimes \delta_{j}\right)=\sum_{u=j y} s s^{i} \otimes \delta_{j} \otimes s \otimes \delta_{y} .
\end{aligned}
$$

For Definition 3.1(iv), where $j \in J_{M}$ and $n \in J_{G}$,

$$
\begin{aligned}
\cdot(\eta \otimes i d)\left(\left(j \otimes \delta_{n}\right) \otimes\left(s \otimes \delta_{u}\right)\right) & =\cdot\left(\sum_{n=z z^{i}}\left(j \otimes \delta_{z}\right) \otimes\left(s \otimes \delta_{u}\right)\right) \\
& =\sum_{n=z z^{i}} \delta_{z, s \triangleright u} j s \otimes \delta_{u}=\sum_{n=u u^{i}} j s \otimes \delta_{u}, \\
\cdot(i d \otimes \eta) \tau\left(\left(j \otimes \delta_{n}\right) \otimes\left(s \otimes \delta_{u}\right)\right) & =\cdot(i d \otimes \eta)\left(\left(s \otimes \delta_{u}\right) \otimes\left(j \otimes \delta_{n}\right)\right) \\
& =\cdot\left(\left(s \otimes \delta_{u}\right) \otimes\left(\sum_{n=z z^{i}} j \otimes \delta_{z}\right)\right)=\sum_{n=z z^{i}} \delta_{u, j \triangleright z} s j \otimes \delta_{z} \\
& =\sum_{n=z z^{i}} \delta_{u, z} j s \otimes \delta_{z}=\sum_{n=u u^{i}} j s \otimes \delta_{u} .
\end{aligned}
$$

For Definition 3.1(v),

$$
\begin{aligned}
\cdot(S \otimes i d) \Delta\left(s \otimes \delta_{u}\right) & =\cdot(S \otimes i d)\left(\sum_{x y=u} s \otimes \delta_{x} \otimes(s \triangleleft x) \otimes \delta_{y}\right) \\
& =\sum_{x y=u}\left((s \triangleleft x)^{i} \otimes \delta_{(s \triangleright x)^{i}}\right)\left((s \triangleleft x) \otimes \delta_{y}\right) \\
& =\sum_{x y=u} \delta_{(s \triangleright x)^{i},(s \triangleleft x) \triangleright y}(s \triangleleft x)^{i}(s \triangleleft x) \otimes \delta_{y} \\
& =\sum_{x y=u} \delta_{(s \triangleright x)^{i},(s \triangleleft x) \triangleright y} s^{i} s \otimes \delta_{y} \\
& =\sum_{x y=u} \delta_{x^{i}, y} s^{i} s \otimes \delta_{y}=\sum_{y^{i} y=u} s^{i} s \otimes \delta_{y},
\end{aligned}
$$


where we have used Proposition 4.4 on (4.15), and applied $(s \triangleleft x)^{i} \triangleright$ to both elements in $\delta_{(s \triangleright x)^{i},(s \triangleleft x) \triangleright y}$ in (4.16).

$$
\begin{aligned}
\cdot(i d \otimes S) \Delta\left(s \otimes \delta_{u}\right) & =\cdot(i d \otimes S)\left(\sum_{x y=u} s \otimes \delta_{x} \otimes(s \triangleleft x) \otimes \delta_{y}\right) \\
& =\sum_{x y=u}\left(s \otimes \delta_{x}\right)\left((s \triangleleft x y)^{i} \otimes \delta_{((s \triangleleft x) \triangleright y)^{i}}\right) \\
& =\sum_{x y=u} \delta_{x,((s \triangleleft x) \triangleleft y)^{i} \triangleright((s \triangleleft x) \triangleright y)^{i}} S(s \triangleleft x y)^{i} \otimes \delta_{((s \triangleleft x) \triangleright y)^{i}} \\
& =\sum_{x y=u} \delta_{x, y^{i}} S(s \triangleleft x y)^{i} \otimes \delta_{((s \triangleleft x) \triangleright y)^{i}} \\
& =\sum_{x y=u} \delta_{x, y^{i}} S S^{i} \otimes \delta_{((s \triangleleft x) \triangleright y)^{i}} \\
& =\sum_{y^{i} y=u} s s^{i} \otimes \delta_{((s \triangleleft y) \triangleright y)^{i}} .
\end{aligned}
$$

In (4.17), we have used the fact that $u=x y=y^{i} y \in J_{G}$. Now change variable in the sum from $y$ to $z^{i}=\left(s \triangleleft y^{i}\right) \triangleright y$. Then $\left(\left(s \triangleleft y^{i}\right) \triangleleft y\right)^{i} \triangleright\left(\left(s \triangleleft y^{i}\right) \triangleright y\right)^{i}=y^{i}$, so $s^{i} \triangleright z=y^{i}$. Then the condition on the summation is $u=y y^{i}=\left(s^{i} \triangleright z\right)^{i}\left(s^{i} \triangleright z\right)=z^{i} z$. This shows that the sums are the same, and that they have value $\eta\left(\epsilon\left(s \otimes \delta_{u}\right)\right)$.

To show that $\Delta$ is an algebra map,

$$
\begin{aligned}
\Delta\left(\left(s \otimes \delta_{u}\right)\left(t \otimes \delta_{v}\right)\right) & =\Delta\left(\delta_{u, t \triangleright v} s t \otimes \delta_{v}\right) \\
& =\sum_{x y=v} \delta_{u, t \triangleright v} s t \otimes \delta_{x} \otimes s t \triangleleft x \otimes \delta_{y} \\
& =\sum_{x y=v=t^{i} \triangleright u} s t \otimes \delta_{x} \otimes s t \triangleleft x \otimes \delta_{y}, \\
\Delta\left(s \otimes \delta_{u}\right) \Delta\left(t \otimes \delta_{v}\right) & =\left(\sum_{x y=u} s \otimes \delta_{x} \otimes s \triangleleft x \otimes \delta_{y}\right)\left(\sum_{x_{1} y_{1}=v} t \otimes \delta_{x_{1}} \otimes t \triangleleft x_{1} \otimes \delta_{y_{1}}\right) \\
& =\sum_{x y=u, x_{1} y_{1}=v}\left(s \otimes \delta_{x}\right)\left(t \otimes \delta_{x_{1}}\right) \otimes\left(s \triangleleft x \otimes \delta_{y}\right)\left(t \triangleleft x_{1} \otimes \delta_{y_{1}}\right) \\
& =\sum_{x y=u, x_{1} y_{1}=v} \delta_{x, t \triangleright x_{1}} \delta_{y,\left(t \triangleleft x_{1}\right) \triangleright y_{1}} s t \otimes \delta_{x_{1}} \otimes(s \triangleleft x)\left(t \triangleleft x_{1}\right) \otimes \delta_{y_{1}} \\
& =\sum_{x y=u, x_{1} y_{1}=v} \delta_{x, t \triangleright x_{1}} \delta_{y,\left(t \triangleleft x_{1}\right) \triangleright y_{1}} s t \otimes \delta_{x_{1}} \otimes s t \triangleleft x_{1} \otimes \delta_{y_{1}} \\
& =\sum_{x_{1} y_{1}=v=t^{i} \triangleright u} s t \otimes \delta_{x_{1}} \otimes s t \triangleleft x_{1} \otimes \delta_{y_{1}} .
\end{aligned}
$$

To show that $\epsilon$ is an algebra map, if $u, v \in J_{G}$,

$$
\begin{aligned}
\epsilon\left(s \otimes \delta_{u}\right) \epsilon\left(t \otimes \delta_{v}\right) & =\left(s s^{i} \otimes \delta_{u}\right)\left(t t^{i} \otimes \delta_{v}\right)=\delta_{u, t t^{i} \triangleright v} s s^{i} t t^{i} \otimes \delta_{v} \\
& =\delta_{u, v} s t t^{i} s^{i} \otimes \delta_{v}=\delta_{u, v}(s t)(s t)^{i} \otimes \delta_{v} \\
\epsilon\left(\left(s \otimes \delta_{u}\right)\left(t \otimes \delta_{v}\right)\right) & =\epsilon\left(\delta_{u, t \triangleright v} s t \otimes \delta_{v}\right)=\delta_{u, v}(s t)(s t)^{i} \otimes \delta_{v} .
\end{aligned}
$$


If $u \notin J_{G}$ or $v \notin J_{G}$ then both expressions give zero. To show that $\eta$ is an algebra map,

$$
\begin{aligned}
\eta\left(j \otimes \delta_{n}\right) \eta\left(j_{1} \otimes \delta_{n_{1}}\right) & =\left(\sum_{z z^{i}=n} j \otimes \delta_{z}\right)\left(\sum_{x x^{i}=n_{1}} j_{1} \otimes \delta_{x}\right) \\
& =\sum_{z z^{i}=n, x x^{i}=n_{1}} \delta_{z, j_{1} \triangleright x} j j_{1} \otimes \delta_{x} \\
& =\sum_{z z^{i}=n, x x^{i}=n_{1}} \delta_{z, x} j j_{1} \otimes \delta_{x} \\
& =\sum_{z z^{i=n}=n_{1}} j j_{1} \otimes \delta_{z}, \\
\eta\left(\left(j \otimes \delta_{n}\right)\left(j_{1} \otimes \delta_{n_{1}}\right)\right) & =\eta\left(\delta_{n, j_{1} \triangleright n_{1}} j j_{1} \otimes \delta_{n_{1}}\right) \\
& =\sum_{z z^{i=n_{1}}} \delta_{n, n_{1}} j j_{1} \otimes \delta_{z} .
\end{aligned}
$$

Proposition 4.6. The map $S$ reverses the order of the product and coproduct.

Proof. For the product,

$$
\begin{aligned}
S\left(\left(s \otimes \delta_{u}\right)\left(t \otimes \delta_{v}\right)\right) & =S\left(\delta_{u, t \triangleright v} s t \otimes \delta_{v}\right)=\delta_{u, t \triangleright v}(s t \triangleleft v)^{i} \otimes \delta_{(s t \triangleright v)^{i}} \\
& =\delta_{u, t \triangleright v}((s \triangleleft(t \triangleright v))(t \triangleleft v))^{i} \otimes \delta_{(s \triangleright(t \triangleright v))^{i}} \\
& =\delta_{u, t \triangleright v}((s \triangleleft u)(t \triangleleft v))^{i} \otimes \delta_{(s \triangleright u)^{i}} \\
& =\delta_{u, t \triangleright v}(t \triangleleft v)^{i}(s \triangleleft u)^{i} \otimes \delta_{(s \triangleright u)^{i}}, \\
S\left(t \otimes \delta_{v}\right) S\left(s \otimes \delta_{u}\right) & =\left((t \triangleleft v)^{i} \otimes \delta_{(t \triangleright v)^{i}}\right)\left((s \triangleleft u)^{i} \otimes \delta_{(s \triangleright u)^{i}}\right) \\
& =\delta_{(t \triangleright v)^{i},(s \triangleleft u)^{i} \triangleright(s \triangleright u)^{i}}(t \triangleleft v)^{i}(s \triangleleft u)^{i} \otimes \delta_{(s \triangleright u)^{i}} \\
& =\delta_{(t \triangleright v)^{i}, u^{i}}(t \triangleleft v)^{i}(s \triangleleft u)^{i} \otimes \delta_{(s \triangleright u)^{i}} .
\end{aligned}
$$

For the coproduct,

$$
\begin{aligned}
\tau \Delta S\left(s \otimes \delta_{u}\right) & =\tau \Delta\left((s \triangleleft u)^{i} \otimes \delta_{(s \triangleright u)^{i}}\right) \\
& =\sum_{x y=(s \triangleright u)^{i}}(s \triangleleft u)^{i} \triangleleft x \otimes \delta_{y} \otimes(s \triangleleft u)^{i} \otimes \delta_{x}, \\
(S \otimes S) \Delta\left(s \otimes \delta_{u}\right) & =\sum_{v w=u}(s \triangleleft v)^{i} \otimes \delta_{(s \triangleright v)^{i}} \otimes(s \triangleleft u)^{i} \otimes \delta_{((s \triangleleft v) \triangleright w)^{i}} .
\end{aligned}
$$

Now we set $x=((s \triangleleft v) \triangleright w)^{i}$ and $y=(s \triangleright v)^{i}$ in the first expression, and observe that we get the second expression.

Proposition 4.7. Let $S_{J}$ be the restriction of $S$ to $H_{J}$, which is just $S_{J}\left(j \otimes \delta_{n}\right)=$ $j^{i} \otimes \delta_{n^{i}}$. Then $\epsilon S=S_{J} \epsilon$ and $S \eta=\eta S_{J}$.

Proof. This is fairly simple, and is left to the reader. 
5. The meromorphic loop group. In this section, we continue with the meromorphic loops introduced in Example 2.3. Any invertible meromorphic function $\phi: \mathbb{C}_{\infty} \rightarrow$ $M_{n}(\mathbb{C})$ which is unitary on the real axis can be written as a constant matrix times a product of factors of the form

$$
\Phi_{\alpha, P}(\lambda)=P^{\perp}+\frac{\lambda-\bar{\alpha}}{\lambda-\alpha} P,
$$

where $\alpha \in \mathbb{C} \backslash \mathbb{R}$ and $P$ is a self-adjoint projection in $M_{n}(\mathbb{C})$. Define the sets

$$
\begin{gathered}
G=\{\phi: \phi(\infty)=1, \phi(\lambda) \text { has no singularities for } \operatorname{im}(\lambda)>0\}, \\
J_{G}=\{\phi: \phi(\infty)=1, \phi(\lambda) \text { has no singularities for } \operatorname{im}(\lambda)>0, \\
\phi \text { is a scalar function times the identity matrix }\}, \\
M=\{\phi: \phi(\infty)=1, \phi(\lambda) \text { has no singularities for } \operatorname{im}(\lambda)<0\}, \\
J_{M}=\{\phi: \phi(\infty)=1, \phi(\lambda) \text { has no singularities for } \operatorname{im}(\lambda)<0, \\
\phi \text { is a scalar function times the identity matrix }\} .
\end{gathered}
$$

The normalization $\phi(\infty)=1$ just means that we can forget about the constant factor. Define the $i$ operation by

$$
\Phi_{\alpha, P}^{i}(\lambda)=\frac{\lambda-\bar{\alpha}}{\lambda-\alpha} P^{\perp}+P,
$$

and extend this to products of basic loops by reversing order, that is, $(\Phi \Psi)^{i}=\Psi^{i} \Phi^{i}$. It is not too difficult to show that $\left(G, J_{G}\right)$ and $\left(M, J_{M}\right)$ are almost groups, with the usual matrix multiplication.

DEFINITION 5.1. We define the actions $\triangleright$ and $\triangleleft$ by reversal of order of multiplication, that is, for $s \in M$ and $u \in G$ choose $s \triangleright u \in G$ and $s \triangleleft u \in M$ so that $s u=(s \triangleright u)(s \triangleleft u)$.

Here we must issue a warning; there is no uniqueness of factorisation. To factor a meromorphic loop $\phi$ we can use the procedure in [1] to write $\phi$ as a product of basic loops, choosing the lower half-plane poles first, $\phi=s u$. There are other possible factorisations of the form $\phi=s^{\prime} u^{\prime}$, where $s^{\prime}=s t \in M$ and $u^{\prime}=t^{-1} u \in G$, all we have to do is to take $t$ with all poles in the upper half-plane, so $t^{-1}$ has all poles in the lower half-plane. This occurs because $G$ and $M$ are not groups, as they are not closed under the inverse operation. To get round this, we always choose a factorisation with the minimum number of basic factors. It is also possible to have ambiguities in the factorisation where poles coincide or are at complex conjugate positions (as noted in the proof of the following proposition). Strictly we should restrict our results on actions to the dense open set of loops which have no multiple poles or poles at complex conjugate positions. We will assume this for the rest of the section (with the exception of the next proposition).

We can calculate the actions on the basic factors by the next result. The actions on products of basic factors are calculated by successive reversals of factors, a procedure which does not increase the number of factors. In fact, $s \triangleright u$ has exactly the same pole positions as $u$, and $s \triangleleft u$ has exactly the same pole positions as $s$. 
Proposition 5.2. Suppose that $\theta_{\alpha}(\lambda)=(\lambda-\bar{\alpha}) /(\lambda-\alpha)$ and $\theta_{\beta}(\lambda)=(\lambda-\bar{\beta}) /(\lambda-\beta)$, where $\alpha$ and $\beta$ are in different half-planes (in particular $\alpha \neq \beta$ ). Then

$$
\left(P_{1}^{\perp}+\theta_{\alpha} P_{1}\right)\left(P_{2}^{\perp}+\theta_{\beta} P_{2}\right)=\left(P_{3}^{\perp}+\theta_{\beta} P_{3}\right)\left(P_{4}^{\perp}+\theta_{\alpha} P_{4}\right),
$$

where (if we put $V_{i}$ to be the image of the projection $P_{i}$ )

$$
V_{3}=\left(P_{1}^{\perp}+\theta_{\alpha}(\beta) P_{1}\right) V_{2}, \quad V_{4}=\left(P_{3}^{\perp}+\theta_{\beta}^{-1}(\alpha) P_{3}\right) V_{1},
$$

if $\beta \neq \bar{\alpha}$, and if $\beta=\bar{\alpha}$ we get $P_{3}=1-P_{1}$ and $P_{4}=1-P_{2}$.

Proof. We know $P_{1}$ and $P_{2}$, and we want to get $P_{3}$ and $P_{4}$. If $\alpha \neq \bar{\beta}$, we have $\left(P_{1}^{\perp}+\right.$ $\left.\theta_{\alpha}(\beta) P_{1}\right) V_{2}=V_{3}$ and $\left(P_{3}^{\perp}+\theta_{\beta}(\alpha) P_{3}\right) V_{4}=V_{1}$, which implies $V_{4}=\left(P_{3}^{\perp}+\theta_{\beta}^{-1}(\alpha) P_{3}\right) V_{1}$. But if $\beta=\bar{\alpha}$ there is a problem, because $\theta_{\beta}(\alpha)$ is not invertible. If $\beta=\bar{\alpha}$, we know that $\theta_{\alpha}(\lambda)=1 / \theta_{\beta}(\lambda)$. Then setting $z=\theta_{\alpha}(\lambda)$, we can write the factorisation as

$$
\left(P_{1}^{\perp}+z P_{1}\right)\left(P_{2}^{\perp}+\frac{1}{z} P_{2}\right)=\left(P_{3}^{\perp}+\frac{1}{z} P_{3}\right)\left(P_{4}^{\perp}+z P_{4}\right),
$$

which can be rearranged to give

$$
\left(P_{2}^{\perp}+\frac{1}{z} P_{2}\right)\left(P_{4}^{\perp}+\frac{1}{z} P_{4}\right)=\left(P_{1}^{\perp}+\frac{1}{z} P_{1}\right)\left(P_{3}^{\perp}+\frac{1}{z} P_{3}\right) .
$$

By separating powers of $z$ we get $P_{4}=P_{3}+P_{1}-P_{2}$ and $\left(P_{1}-P_{2}\right) P_{3}=P_{2}\left(P_{1}-P_{2}\right)$. In the case where $P_{1}-P_{2}$ is invertible, we can define $P_{3}$ as the unique solution to $\left(P_{1}-P_{2}\right) P_{3}=P_{2}\left(P_{1}-P_{2}\right)$, and this will then give a unique value of $P_{4}$. From substituting in the equation we see that these unique solutions are $P_{3}=1-P_{1}$ and $P_{4}=1-P_{2}$. To preserve continuity, we define these to be the actions even if $P_{1}-P_{2}$ is not invertible.

Proposition 5.3. The meromorphic loop almost groups $\left(G, J_{G}\right)$ and $\left(M, J_{M}\right)$, with the actions and $i$ operation specified, form a matched pair.

Proof. Consider the associativity of the multiplication stu where $s, t \in M$ and $u \in G$. Then,

$$
\begin{aligned}
s(t u) & =(s t) u=(s t \triangleright u)(s t \triangleleft u)=s(t \triangleright u)(t \triangleleft u) \\
& =(s \triangleright(t \triangleright u))(s \triangleleft(t \triangleright u))(t \triangleleft u) .
\end{aligned}
$$

By the uniqueness of the factorisation (on the open dense subset referred to earlier), we see that $s \triangleright(t \triangleright u)=s t \triangleright u$ and $(s \triangleleft(t \triangleright u))(t \triangleleft u)=s t \triangleleft u$. Similarly, for all $s \in M$ and $u, v \in G$, we have

$$
\begin{aligned}
(s u) v=s(u v) & =(s \triangleright u v)(s \triangleleft u v)=(s \triangleright u)(s \triangleleft u) v \\
& =(s \triangleright u)((s \triangleleft u) \triangleright v)((s \triangleleft u) \triangleleft v)
\end{aligned}
$$

which gives $(s \triangleright u)((s \triangleleft u) \triangleright v)=s \triangleright u v$ and $(s \triangleleft u) \triangleleft v=s \triangleleft u v$. Also, for all $j \in J_{M}$ and $u \in G$, we have $j u=u j=(j \triangleright u)(j \triangleleft u)$, which gives $j \triangleright u=u$ and $j \triangleleft u=j$. 
Similarly, for all $j \in J_{G}$ and $s \in M$, we have $s j=j s=(s \triangleright j)(s \triangleleft j)$, which gives $s \triangleright j=j$ and $s \triangleleft j=s$. Finally, for all $s \in M$ and $u \in G$, we have

$$
\begin{aligned}
(s u)^{i} & =u^{i} s^{i}=((s \triangleright u)(s \triangleleft u))^{i}=(s \triangleleft u)^{i}(s \triangleright u)^{i} \\
& =\left((s \triangleleft u)^{i} \triangleright(s \triangleright u)^{i}\right)\left((s \triangleleft u)^{i} \triangleleft(s \triangleright u)^{i}\right) .
\end{aligned}
$$

By the uniqueness of the factorisation, we see that

$$
(s \triangleleft u)^{i} \triangleright(s \triangleright u)^{i}=u^{i}, \quad(s \triangleleft u)^{i} \triangleleft(s \triangleright u)^{i}=s^{i} .
$$

6. Duality. We take $\left(G, J_{G}\right)$ and $\left(M, J_{M}\right)$ to be a matched pair of finite almost groups. There is a dual almost Hopf algebra $H^{\prime}=k(M) \bowtie k G$ to $H=k M \bowtie k(G)$ with basis $\delta_{s} \otimes u$ where $s \in M$ and $u \in G$, with $J_{H^{\prime}}=k\left(J_{M}\right) \bowtie k J_{G}$. The explicit formulae for this almost Hopf algebra are as follows:

$$
\begin{aligned}
& \left(\delta_{s} \otimes u\right)\left(\delta_{t} \otimes v\right)=\delta_{s \triangleleft u, t}\left(\delta_{s} \otimes u v\right), \quad \Delta\left(\delta_{s} \otimes u\right)=\sum_{a, b \in M: a b=s} \delta_{a} \otimes b \triangleright u \otimes \delta_{b} \otimes u, \\
& S\left(\delta_{s} \otimes u\right)=\delta_{(s \triangleleft u)^{i}} \otimes(s \triangleright u)^{i}, \quad \epsilon\left(\delta_{s} \otimes u\right)= \begin{cases}0, & \text { if } s \notin J_{M}, \\
\delta_{s} \otimes u u^{i}, & \text { if } s \in J_{M},\end{cases} \\
& \eta\left(\delta_{j} \otimes n\right)=\sum_{a \in M: j=a a^{i}} \delta_{a} \otimes n .
\end{aligned}
$$

The dual pairing between $H^{\prime}$ and $H$ is given by

$$
\left\langle\delta_{s} \otimes u, t \otimes \delta_{v}\right\rangle=\delta_{s, t} \delta_{u, v}
$$

Proposition 6.1. The almost Hopf algebras $H=k M \bowtie k(G)$ and $H^{\prime}=k(M) \bowtie k G$ are dual to each other.

PROoF. First we check that the counits and the units are dual to each other:

$$
\begin{aligned}
& \left\langle\epsilon\left(\delta_{s} \otimes u\right), j \otimes \delta_{n}\right\rangle=\delta_{s, j} \delta_{u u^{i}, n} \\
& \left\langle\delta_{s} \otimes u, \eta\left(j \otimes \delta_{n}\right)\right\rangle=\left\langle\delta_{s} \otimes u, \sum_{n=z z^{i}} j \otimes \delta_{z}\right\rangle=\delta_{s, j} \delta_{u u^{i}, n}, \\
& \left\langle\delta_{j} \otimes n, \epsilon\left(s \otimes \delta_{u}\right)\right\rangle=\delta_{j, s s^{i}} \delta_{n, u} \\
& \left\langle\eta\left(\delta_{j} \otimes n\right), s \otimes \delta_{u}\right\rangle=\left\langle\sum_{j=z z^{i}} \delta_{z} \otimes n, s \otimes \delta_{u}\right\rangle=\delta_{j, s s^{i}} \delta_{n, u} .
\end{aligned}
$$

Now we check the antipodes

$$
\begin{aligned}
& \left\langle S\left(\delta_{s} \otimes u\right), t \otimes \delta_{v}\right\rangle=\left\langle\delta_{(s \triangleleft u)^{i}} \otimes(s \triangleright u)^{i}, t \otimes \delta_{v}\right\rangle=\delta_{(s \triangleleft u)^{i}, t} \delta_{(s \triangleright u)^{i}, v}, \\
& \left\langle\delta_{s} \otimes u, S\left(t \otimes \delta_{v}\right)\right\rangle=\left\langle\delta_{s} \otimes u,(t \triangleleft v)^{i} \otimes \delta_{(t \triangleright v)^{i}}\right\rangle=\delta_{s,(t \triangleleft v)^{i}} \delta_{u,(t \triangleright v)^{i}},
\end{aligned}
$$

and these are the same by the original definition of the actions. It is left to the reader 
to check the product and coproduct, that is,

$$
\begin{aligned}
& \left\langle\left(\delta_{s} \otimes u\right)\left(\delta_{t} \otimes v\right), r \otimes \delta_{w}\right\rangle=\left\langle\left(\delta_{s} \otimes u\right) \otimes\left(\delta_{t} \otimes v\right), \Delta\left(r \otimes \delta_{w}\right)\right\rangle, \\
& \left\langle\delta_{s} \otimes u,\left(t \otimes \delta_{v}\right)\left(r \otimes \delta_{w}\right)\right\rangle=\left\langle\Delta\left(\delta_{s} \otimes u\right),\left(t \otimes \delta_{v}\right) \otimes\left(r \otimes \delta_{w}\right)\right\rangle .
\end{aligned}
$$

7. The $*$ operation. We take $\left(G, J_{G}\right)$ and $\left(M, J_{M}\right)$ to be a matched pair of finite almost groups, and continue with the notation of the last section. We define a $*$ operation on $H$ by $\left(s \otimes \delta_{u}\right)^{*}=s^{i} \otimes \delta_{s \triangleright u}$ on the basis elements, extended to a conjugate-linear map from $H$ to $H$.

Proposition 7.1. The $*$ operation reverses the order of multiplication.

Proof. Applying the $*$ map to a product,

$$
\begin{aligned}
\left(\left(s \otimes \delta_{u}\right)\left(t \otimes \delta_{v}\right)\right)^{*} & =\left(\delta_{u, t \triangleright v} s t \otimes \delta_{v}\right)^{*} \\
& =\delta_{u, t \triangleright v}(s t)^{i} \otimes \delta_{s t \triangleright v}, \\
\left(t \otimes \delta_{v}\right)^{*}\left(s \otimes \delta_{u}\right)^{*} & =\left(t^{i} \otimes \delta_{t \triangleright v}\right)\left(s^{i} \otimes \delta_{s \triangleright u}\right) \\
& =\delta_{t \triangleright v, s^{i} \triangleright(s \triangleright u)} t^{i} s^{i} \otimes \delta_{s \triangleright u} \\
& =\delta_{t \triangleright v, s^{i} s \triangleright u}(s t)^{i} \otimes \delta_{s \triangleright u} \\
& =\delta_{t \triangleright v, u}(s t)^{i} \otimes \delta_{s \triangleright(t \triangleright v)} .
\end{aligned}
$$

Proposition 7.2. The $*$ operation preserves the comultiplication.

Proof. Applying the $*$ map to a coproduct,

$$
\begin{aligned}
\Delta\left(\left(s \otimes \delta_{u}\right)^{*}\right) & =\Delta\left(s^{i} \otimes \delta_{s \triangleright u}\right) \\
& =\sum_{x y=s \triangleright u} s^{i} \otimes \delta_{x} \otimes s^{i} \triangleleft x \otimes \delta_{y}, \\
\left(\Delta\left(s \otimes \delta_{u}\right)\right)^{*} & =\left(\sum_{x_{1} y_{1}=u} s \otimes \delta_{x_{1}} \otimes s \triangleleft x_{1} \otimes \delta_{y_{1}}\right)^{*} \\
& =\sum_{x_{1} y_{1}=u} s^{i} \otimes \delta_{s \triangleright x_{1}} \otimes\left(s \triangleleft x_{1}\right)^{i} \otimes \delta_{\left(s \triangleleft x_{1}\right) \triangleright y_{1}} .
\end{aligned}
$$

Since $s \triangleright u=s \triangleright x_{1} y_{1}=\left(s \triangleright x_{1}\right)\left(\left(s \triangleleft x_{1}\right) \triangleright y_{1}\right)$, if we consider $x=s \triangleright x_{1}$ (i.e., $\left.x_{1}=s^{i} \triangleright x\right)$ and $y=\left(s \triangleleft x_{1}\right) \triangleright y_{1}$ (i.e., $\left.y_{1}=\left(s \triangleleft x_{1}\right)^{i} \triangleright y\right)$ we see that the two sums are the same.

Proposition 7.3. The $*$ operation preserves the unit, counit, and antipode.

Proof. For the unit,

$$
\begin{aligned}
& \left(\eta\left(j \otimes \delta_{n}\right)\right)^{*}=\left(\sum_{z z^{i}=n} j \otimes \delta_{z}\right)^{*}=\sum_{z z^{i}=n} j^{i} \otimes \delta_{j \triangleright z}=\sum_{z z^{i}=n} j^{i} \otimes \delta_{z}, \\
& \eta\left(\left(j \otimes \delta_{n}\right)^{*}\right)=\eta\left(j^{i} \otimes \delta_{j \triangleright n}\right)=\sum_{z z^{i}=j \triangleright n} j^{i} \otimes \delta_{z}=\sum_{z z^{i}=n} j^{i} \otimes \delta_{z} .
\end{aligned}
$$


For the counit,

$$
\begin{aligned}
\epsilon\left(\left(s \otimes \delta_{u}\right)^{*}\right) & =\epsilon\left(s^{i} \otimes \delta_{s \triangleright u}\right) \\
& = \begin{cases}0, & \text { if } s \triangleright u \notin J_{G} \\
s^{i} s \otimes \delta_{s \triangleright u}, & \text { if } s \triangleright u \in J_{G}\end{cases} \\
& = \begin{cases}0, & \text { if } u \notin J_{G} \\
s^{i} s \otimes \delta_{u}, & \text { if } u \in J_{G}\end{cases} \\
& =\left(\epsilon\left(s \otimes \delta_{u}\right)\right)^{*} .
\end{aligned}
$$

For the antipode,

$$
\begin{aligned}
\left(S\left(s \otimes \delta_{u}\right)\right)^{*} & =\left((s \triangleleft u)^{i} \otimes \delta_{(s \triangleright u)^{i}}\right)^{*} \\
& =\left((s \triangleleft u)^{i}\right)^{i} \otimes \delta_{(s \triangleleft u)^{i} \triangleright(s \triangleright u)^{i}} \\
& =(s \triangleleft u) \otimes \delta_{u^{i}}, \\
S\left(\left(s \otimes \delta_{u}\right)^{*}\right) & =S\left(s^{i} \otimes \delta_{s \triangleright u}\right) \\
& =\left(s^{i} \triangleleft(s \triangleright u)\right)^{i} \otimes \delta_{\left(s^{i} \triangleright(s \triangleright u)\right)^{i}} \\
& =\left(s^{i} \triangleleft(s \triangleright u)\right)^{i} \otimes \delta_{\left(s^{i} s \triangleright u\right)^{i}} \\
& =(s \triangleleft u) \otimes \delta_{u^{i}},
\end{aligned}
$$

as $s^{i} \triangleleft(s \triangleright u)=(s \triangleleft u)^{i}$ (just apply $\triangleleft(s \triangleright u)^{i}$ to both sides).

8. Mutually inverse matched pairs. Here we discuss a property motivated by the meromorphic loop example discussed earlier.

DEFINITION 8.1. The matched pair $\left(G, J_{G}\right)$ and $\left(M, J_{M}\right)$ is said to be mutually inverse if the following conditions hold:

(i) the doublecross product $G \bowtie M$ is a group, with identity $e=\left(e_{G}, e_{M}\right) \in J_{G} \times J_{M}$ and inverse operation $x \mapsto x^{-1}$;

(ii) for all $s \in M, s^{-1} \in G$ and also for all $u \in G, u^{-1} \in M$;

(iii) the map inverse: $J_{G} \rightarrow J_{M}$ is a 1-1 correspondence;

(iv) for all $x \in G \bowtie M,\left(x^{-1}\right)^{i}=\left(x^{i}\right)^{-1}$;

(v) for all $s \in M$ and $u \in G, u^{-1} \triangleright s^{-1}=(s \triangleleft u)^{-1}$ and $u^{-1} \triangleleft s^{-1}=(s \triangleright u)^{-1}$.

Note that from Definition 8.1(i) it can easily be seen that $e_{G}$ is an identity for $G$, and that $e_{M}$ is an identity for $M$. We can then imbed $G \subset G \bowtie M$ by $u \mapsto\left(u, e_{M}\right)$ and $M \subset G \bowtie M$ by $s \mapsto\left(e_{G}, s\right)$. Then Definition 8.1(ii) can more properly be written as $\left(u, e_{M}\right)^{-1}=\left(e_{G}, u^{-1}\right)$ and $\left(e_{G}, s\right)^{-1}=\left(s^{-1}, e_{M}\right)$.

EXAMPLE 8.2. The meromorphic loop almost groups defined in Definition 5.1 form a mutually inverse matched pair (with the usual caveat about densely defined actions).

The doublecross product just consists of meromorphic loops which are unitary on the real axis, with the usual pointwise multiplication. On the single pole factors the 
inverse is

$$
\left(P^{\perp}+\frac{\lambda-\bar{\alpha}}{\lambda-\alpha} P\right)^{-1}=P^{\perp}+\frac{\lambda-\alpha}{\lambda-\bar{\alpha}} P,
$$

so that a factor with a pole in the upper half-plane has an inverse with a pole in the lower half-plane, and vice versa. It is fairly easy to check that $\left(x^{-1}\right)^{i}=\left(x^{i}\right)^{-1}$ from this formula.

If we take a factorisation $s u=(s \triangleright u)(s \triangleleft u)$ (where $s \in M$ and $u \in G$ ), and take the inverses of both sides we get $u^{-1} s^{-1}=(s \triangleleft u)^{-1}(s \triangleright u)^{-1}$. But $u^{-1} \in M$ and $s^{-1} \in G$, so $u^{-1} s^{-1}=\left(u^{-1} \triangleright s^{-1}\right)\left(u^{-1} \triangleleft s^{-1}\right)$. As both $u^{-1} \triangleright s^{-1}$ and $(s \triangleleft u)^{-1}$ have the same pole positions we see that $u^{-1} \triangleright s^{-1}=(s \triangleleft u)^{-1}$, and similarly, $u^{-1} \triangleleft s^{-1}=(s \triangleright u)^{-1}$.

DEFINITION 8.3. In the case where we have a mutually inverse matched pair of finite almost groups, we define the map $T: H=k M \bowtie k(G) \rightarrow H^{\prime}=k(M) \bowtie k G$ by $T\left(s \otimes \delta_{u}\right)=\delta_{u^{-1}} \otimes s^{-1}$, and $T_{J}: J_{H} \rightarrow J_{H^{\prime}}$ by $T_{J}\left(j \otimes \delta_{n}\right)=\delta_{n^{-1}} \otimes j^{-1}$.

Proposition 8.4. The map $T$ reverses the order of both multiplication and comultiplication.

Proof. For multiplication,

$$
\begin{aligned}
T\left(\left(s \otimes \delta_{u}\right)\left(t \otimes \delta_{v}\right)\right) & =T\left(\delta_{u, t \triangleright v} s t \otimes \delta_{v}\right)=\delta_{u, t \triangleright v} \delta_{v^{-1}} \otimes(s t)^{-1} \\
T\left(t \otimes \delta_{v}\right) T\left(s \otimes \delta_{u}\right) & =\left(\delta_{v^{-1}} \otimes t^{-1}\right)\left(\delta_{u^{-1}} \otimes s^{-1}\right)=\delta_{v^{-1} \triangleleft t^{-1}, u^{-1}} \delta_{v^{-1}} \otimes t^{-1} s^{-1} \\
& =\delta_{(t \triangleright v)^{-1}, u^{-1}} \delta_{v^{-1}} \otimes(s t)^{-1} .
\end{aligned}
$$

For comultiplication,

$$
\begin{aligned}
(T \otimes T)\left(\Delta\left(s \otimes \delta_{u}\right)\right) & =(T \otimes T)\left(\sum_{x y=u} s \otimes \delta_{x} \otimes s \triangleleft x \otimes \delta_{y}\right) \\
& =\sum_{x y=u} \delta_{x^{-1}} \otimes s^{-1} \otimes \delta_{y^{-1}} \otimes(s \triangleleft x)^{-1}, \\
\tau \Delta\left(T\left(s \otimes \delta_{u}\right)\right) & =\tau \Delta\left(\delta_{u^{-1}} \otimes s^{-1}\right)=\tau\left(\sum_{a b=u^{-1}} \delta_{a} \otimes b \triangleright s^{-1} \otimes \delta_{b} \otimes s^{-1}\right) \\
& =\sum_{a b=u^{-1}} \delta_{b} \otimes s^{-1} \otimes \delta_{a} \otimes b \triangleright s^{-1},
\end{aligned}
$$

which can be seen to be the same on substituting $a=y^{-1}$ and $b=x^{-1}$.

Proposition 8.5. The map $T$ preserves the antipode and $*$-operation, where $*$ on $H^{\prime}$ is defined by $\left(\delta_{s} \otimes u\right)^{*}=\delta_{s \triangleleft u} \otimes u^{i}$.

Proof. For the antipode,

$$
\begin{aligned}
S T\left(s \otimes \delta_{u}\right) & =S\left(\delta_{u^{-1}} \otimes s^{-1}\right)=\delta_{\left(u^{-1} \triangleleft s^{-1}\right)^{i}} \otimes\left(u^{-1} \triangleright s^{-1}\right)^{i} \\
& =\delta_{\left((s \triangleright u)^{-1}\right)^{i}} \otimes\left((s \triangleleft u)^{-1}\right)^{i}, \\
T S\left(s \otimes \delta_{u}\right) & =T\left((s \triangleleft u)^{i} \otimes \delta_{(s \triangleright u)^{i}}\right)=\delta_{\left((s \triangleright u)^{i}\right)^{-1}} \otimes\left((s \triangleleft u)^{i}\right)^{-1} .
\end{aligned}
$$


For the $*$-operation,

$$
\begin{aligned}
& (* \circ T)\left(s \otimes \delta_{u}\right)=*\left(\delta_{u^{-1}} \otimes s^{-1}\right)=\delta_{u^{-1} \triangleleft s^{-1}} \otimes\left(s^{-1}\right)^{i}=\delta_{(s \triangleright u)^{-1}} \otimes\left(s^{-1}\right)^{i}, \\
& (T \circ *)\left(s \otimes \delta_{u}\right)=T\left(s^{i} \otimes \delta_{s \triangleright u}\right)=\delta_{(s \triangleright u)^{-1}} \otimes\left(s^{i}\right)^{-1}=\delta_{(s \triangleright u)^{-1}} \otimes\left(s^{-1}\right)^{i} .
\end{aligned}
$$

Proposition 8.6. The maps $T$ and $T_{J}$ preserve the unit and counit.

Proof. For the unit,

$$
\begin{aligned}
& T \eta\left(j \otimes \delta_{n}\right)=T\left(\sum_{z \in G: z z^{i}=n} j \otimes \delta_{z}\right)=\sum_{z \in G: z z^{i}=n} \delta_{z^{-1}} \otimes j^{-1}, \\
& \eta T_{J}\left(j \otimes \delta_{n}\right)=\eta\left(\delta_{n^{-1}} \otimes j^{-1}\right)=\sum_{a \in M: a a^{i}=n^{-1}} \delta_{a} \otimes j^{-1},
\end{aligned}
$$

and these are the same by putting $a=z^{-1}$.

For the counit, if $u \in J_{G}$,

$$
\begin{aligned}
& \epsilon T\left(s \otimes \delta_{u}\right)=\epsilon\left(\delta_{u^{-1}} \otimes s^{-1}\right)=\delta_{u^{-1}} \otimes s^{-1}\left(s^{-1}\right)^{i}=\delta_{u^{-1}} \otimes\left(s^{i} s\right)^{-1}, \\
& T_{J} \epsilon\left(s \otimes \delta_{u}\right)=T_{J}\left(s s^{i} \otimes \delta_{u}\right)=\delta_{u^{-1}} \otimes\left(s s^{i}\right)^{-1}=\delta_{u^{-1}} \otimes\left(s^{i} s\right)^{-1} .
\end{aligned}
$$

If $u \notin J_{G}$ then both expressions will give zero.

THEOREM 8.7. The almost Hopf algebra $H=k M \triangleright k(G)$ is self dual by the map $H \stackrel{S}{\rightarrow} H \stackrel{T}{\rightarrow} H^{\prime}$.

Proof. We have seen that both $S$ and $T$ reverse the order of the product and coproduct, and preserve the unit, counit, and antipode. Further, both $S$ and $T$ are invertible.

ACKNOWLEDGments. The authors would like to thank T. Brzezinski (Swansea), M. V. Lawson (Bangor), and S. Majid (QMW London) for their assistance, and the referees for several useful suggestions.

\section{REFERENCES}

[1] E. J. Beggs, Solitons in the chiral equation, Comm. Math. Phys. 128 (1990), no. 1, 131-139.

[2] E. J. Beggs, J. D. Gould, and S. Majid, Finite group factorizations and braiding, J. Algebra 181 (1996), no. 1, 112-151.

[3] G. Bòhm and K. Szlachónyi, A coassociative $C^{*}$-quantum group with nonintegral dimensions, Lett. Math. Phys. 38 (1996), no. 4, 437-456.

[4] M. V. Lawson, Inverse Semigroups, World Scientific Publishing, New Jersey, 1998.

[5] S. Majid, Foundations of Quantum Group Theory, Cambridge University Press, Cambridge, 1995.

[6] J. W. Milnor, Construction of universal bundles. I, Ann. of Math. (2) 63 (1956), 272-284.

[7] J. W. Milnor and J. D. Stasheff, Characteristic Classes, Princeton University Press, New Jersey, 1974.

[8] S. Novikov, S. V. Manakov, L. P. Pitaevskiŭ, and V. E. Zakharov, Theory of Solitons, Consultants Bureau [Plenum], New York, 1984.

[9] G. Segal, Classifying spaces and spectral sequences, Inst. Hautes Études Sci. Publ. Math. (1968), no. 34, 105-112. 
[10] M. Takeuchi, Matched pairs of groups and bismash products of Hopf algebras, Comm. Algebra 9 (1981), no. 8, 841-882.

[11] A. B. Zamolodchikov and A. B. Zamolodchikov, Factorized S-matrices in two dimensions as the exact solutions of certain relativistic quantum field theory models, Ann. Physics 120 (1979), no. 2, 253-291.

Falleh R. Al-Solamy: Department of Mathematics, Faculty of Science, King Abdul AZIZ UNIVERSITY, P.O. BOX 80015, JEDDAH 21589, SAUdi ARABIA

EDWin J. Beggs: Department of Mathematics, University of WAles SWANSEa, SWANSEA, SA2 8PP, WALES, UK 


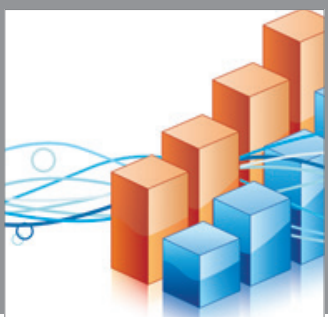

Advances in

Operations Research

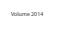

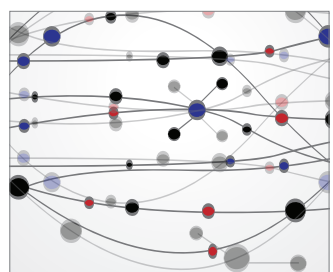

\section{The Scientific} World Journal
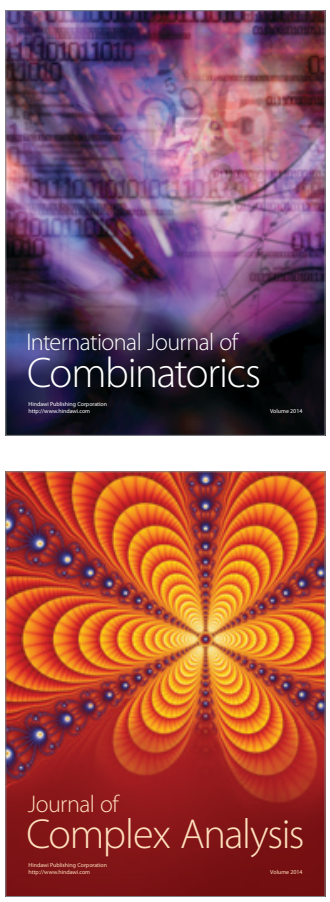

International Journal of

Mathematics and

Mathematical

Sciences
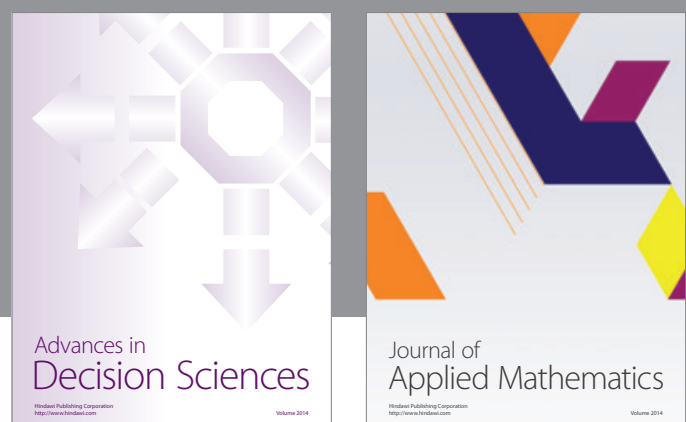

Journal of

Applied Mathematics
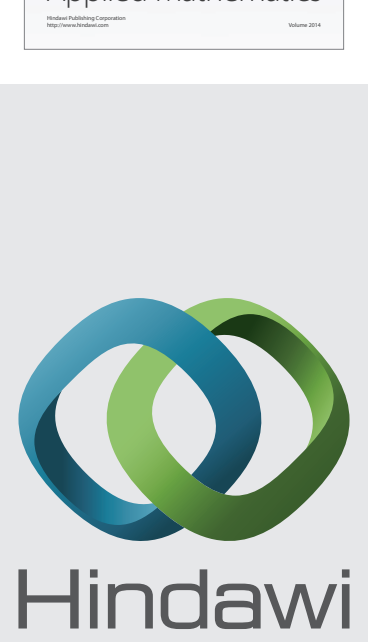

Submit your manuscripts at http://www.hindawi.com
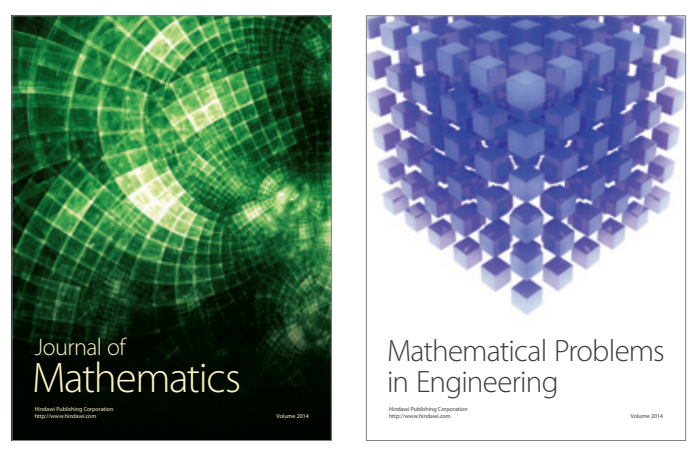

Mathematical Problems in Engineering
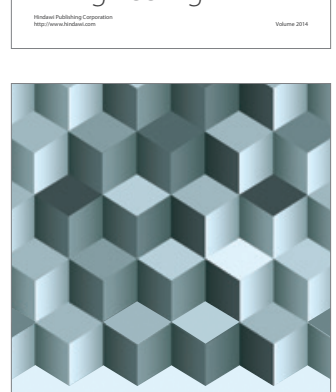

Journal of

Function Spaces
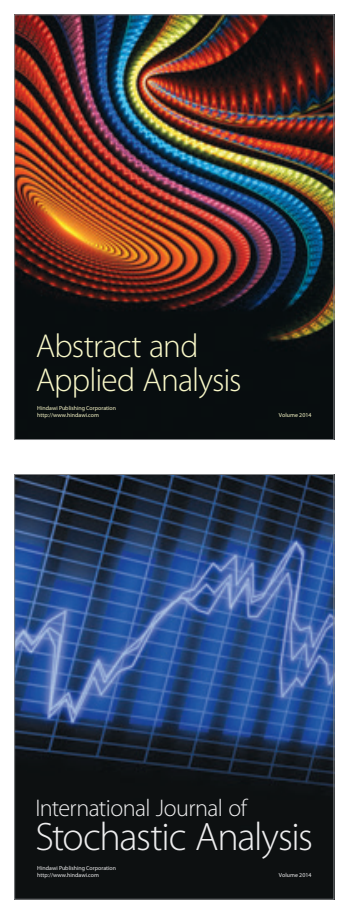

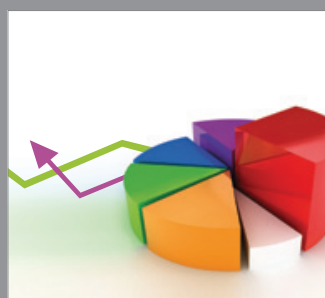

ournal of

Probability and Statistics

Promensencen
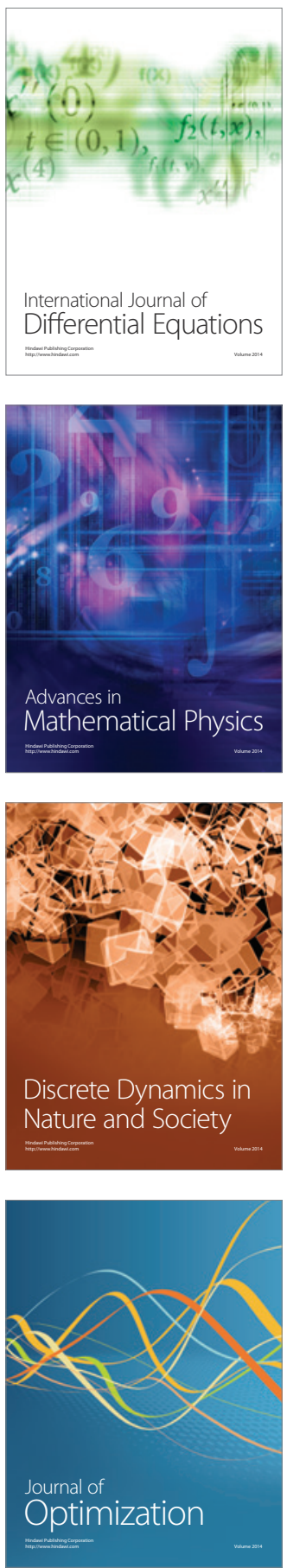